\title{
Macular colour contrast sensitivity in ocular hypertension and glaucoma: evidence for two types of defect
}

\author{
F M Falcao-Reis, F O'Sullivan, W Spileers, C Hogg, G B Arden
}

\begin{abstract}
Colour contrast sensitivity (CCS) of a large cohort of glaucomatous patients, ocular hypertensive patients $(\mathrm{OH})$, and normal persons was measured at six-month intervals during a twoyear period. The OHs were graded into high, medium, and low risk groups. 69\% of glaucomatous patients and 32\% of all OHs had CCS thresholds greater than the mean plus 2 SDs of the controls. Satisfactory specificity and sensitivity could not be obtained by adjusting the criterion of threshold. In abnormal eyes, progressive small increases of threshold occurred during the study, but glaucomatous eyes with normal thresholds on the first visit retained normal thresholds in the subsequent visits. Although our system is very sensitive and precise, the proportion of abnormalities detected is no greater than with other techniques. In some glaucomatous patients there is a true preservation of colour vision which does not merely reflect the limitations of the test employed.
\end{abstract}

Acquired dyschromatopsia can occur early in the course of glaucoma. A blue-yellow loss of colour vision is usually found and may be apparent before nerve fibre bundle defects are detected or visual field defects found. 'In addition it has been shown that some eyes with ocular hypertension $(\mathrm{OH})$ also have a similar defect. ${ }^{23}$ Drance $^{4}$ reported a colour vision loss in $20 \%$ of cases of $\mathrm{OH}$ and suggested that testing colour vision could help to identify those who are likely to develop glaucoma. However, some glaucoma patients never show abnormal colour vision, and conversely some $\mathrm{OH}$ patients who have abnormal colour vision never develop glaucoma. ${ }^{4}$ These discrepancies might either reflect limitations in testing techniques or the complex relation between mechanisms of loss of colour vision and the increase in intraocular pressure (IOP). In principle a distinction could be made between these classes of hypothesis if a more sensitive test of colour vision were to be available.

Colour vision techniques used in clinical practice include the Ishihara test, AO pseudoisochromatic plates, 100-hue Farnsworth and its derivatives, and the anomaloscope ${ }^{5-9}$ (for a review see $\mathrm{Hart}^{10}$ ). Another technique which has been used to assess colour vision changes, especially in patients with glaucoma, is colour perimetry. ${ }^{11-13}$ Such tests tend to confound luminance with colour increments. The latest results suggest this technique is superior in $\mathrm{OH}^{1+}$ to conventional achromatic perimetry.

Colour contrast sensitivity (CCS) measure- ments, recently introduced, ${ }^{5}$ measure true hue discrimination. In CCS the test and the surround have the same luminance and differ only in chromaticity. Such tests have been made possible by recent advances in video and computer technology. In this work we used such a new system which has been fully described elsewhere. ${ }^{16}$ Our system proved that abnormalities can be detected in very mild disease, ${ }^{17}$ and preliminary results suggested that it could discriminate between ocular hypertensive patients and glaucomatous patients and the normal population. ${ }^{18}$ We have tested this hypothesis by measuring colour vision in three different classes of $\mathrm{OH}$ patients classified in ways which are known to separate the groups into differing rates of conversion to glaucoma. ${ }^{19-21}$

\section{Material and methods}

\section{CLINICAL EXAMINATIONS}

Patients' eyes were examined by biomicroscopy, funduscopy, disc photography, applanation tonometry, and Humphrey perimetry, and classified by the usual criteria as suffering from either glaucoma or ocular hypertension. Glaucomatous eyes had a visual defect consisting of one locus of sensitivity loss of $10 \mathrm{~dB}$ or greater flanked by at least one or more adjacent spots of $5 \mathrm{~dB}$ depression. This observation had to be made on at least two occasions. Hypertensive eyes were further classified into three groups by Yablonski's criteria. $^{22}$

\section{COLOUR VISION TESTS}

Colour contrast sensitivity was measured by a system fully described elsewhere. ${ }^{16}$ In brief, a computer-graphics peripheral controls the image on a high quality monitor. A program calculates the relative voltages required to produce any colour (within the limits imposed by the TV phosphors) in terms of the CIE 1931 diagram of colour space. Hues were modulated along colour confusion lines for trichromatic vision. Thus protan, deutan, and tritan 'colour axes' were used. The program produces gratings (or other images) in which there is no spatial variation of luminance, and changes in colour contrast are the only clues to image recognition.

To improve precision, individual variations from the CIE (Commission Internationale d'Eclairage) standard observer are first measured. The patient makes a heterochromatic flicker brightness match between red and green and blue and green TV guns. This match is compared with that of the normal observer. The 
Table 1 Mean tritan thresholds for all classes - first visits

\begin{tabular}{|c|c|c|c|c|}
\hline Class & Age & $\begin{array}{l}\text { Protan } \\
(\%)\end{array}$ & $\begin{array}{l}\text { Deutan } \\
(\%)\end{array}$ & $\begin{array}{l}\text { Tritan } \\
(\%)\end{array}$ \\
\hline \multicolumn{5}{|l|}{ Glaucoma (n=2) } \\
\hline Mean & 57 & 11 & 11 & 19 \\
\hline SD & 14 & 6 & 8 & 16 \\
\hline High risk $(n=59)$ & 56 & 8 & 8 & \\
\hline SD & 10 & 3 & 4 & 4 \\
\hline \multicolumn{3}{|l|}{ Medium risk $(n=56)$} & & \\
\hline $\begin{array}{l}\text { Mean } \\
\text { SD }\end{array}$ & 11 & 3 & 2 & 4 \\
\hline \multicolumn{5}{|l|}{ Low risk $(n=54)$} \\
\hline $\begin{array}{l}\text { Mean } \\
\text { SD }\end{array}$ & 9 & 3 & $\begin{array}{l}8 \\
2\end{array}$ & $\begin{array}{l}9 \\
3\end{array}$ \\
\hline \multicolumn{5}{|l|}{$\operatorname{Control}(n=29)$} \\
\hline $\begin{array}{l}\text { Mean } \\
\text { SD }\end{array}$ & $\begin{array}{l}42 \\
14\end{array}$ & $\begin{array}{l}5 \\
2\end{array}$ & $\begin{array}{l}6 \\
2\end{array}$ & 2 \\
\hline
\end{tabular}

output is then adjusted to produce equiluminous colours for the patient tested, compensating for his lens changes or macular pigmentation. A square wave grating of $0.6 \mathrm{c} /$ degree filling $25 \%$ of the screen was the image. At such a low frequency CCS is not affected by reduced visual acuity. The surround was a mixture of the two colours of which the grating was composed. When colour contrast was 0 , all parts of the grating had the same hue as the background. A maximum contrast of $100 \%$ was arbitrarily defined as most extreme colours in the 'colour axis' displayed. Gratings appeared once a second for $200 \mathrm{~ms}$. Threshold determinations were carried out monocularly by the method of ascending limits. An increment of 0.01 of maximum contrast was used. The computer produced a warning tone when the staircase began. The patient held a 'mouse' and pressed its button when the grating appeared. The colour contrast was then reduced by a randomly selected amount to a subthreshold value and rose again following a new warning tone. At least three determinations were averaged to obtain a threshold. The computer calculated the mean threshold and its standard error.

\section{ELECTROPHYSIOLOGY: PATTERN \\ ELECTRORETINOGRAPHY}

The PERG was recorded in ways described in other reports. ${ }^{23}$ Briefly, a gold foil electrode was used to record from the cornea, and the reference was on the temple. $96 \%$ contrast $30^{\prime}$ squares were used, reversing at $3 \mathrm{~Hz}$. About 1000 responses were averaged.

\section{PATIENTS}

All the patients and normal subjects had a visual acuity of $6 / 6$ or better and did not suffer from any other ocular diseases, particularly cataract or corneal disease. The patients were tested during 24 months at six-monthly intervals. They were part of a cohort who are being investigated in several clinical trials. ${ }^{18+25}$ Normal persons were patients' relatives or hospital staff.

\section{Results}

PATIENTS WITH GLAUCOMA

Thirty two eyes with early glaucoma were tested on the first visit. Twenty four were tested on two occasions, 16 on three, and 10 on four.

The glaucomatous patients' thresholds (obtained for colour contrasts along the three colour confusion lines) on the first visit are shown in Table 1. It can be seen that the mean threshold was increased by comparison with all $\mathrm{OH}$ groups or the control group, the difference being more evident in the tritan axis. Therefore further analysis is restricted to this colour combination. Fig 1 shows the distribution of thresholds in the tritan axis. There was a large overlap in the distribution of thresholds between the glaucoma group and the others, but the distributions for glaucomatous patients and high risk were skewed to higher values, to a much greater extent than for the other groups.

Mean tritan threshold was $19 \%$ for glaucoma and $7 \%$ for normal persons. A large proportion of the glaucomatous eyes $(69 \%)$ deviated from the control group mean by 2 standard deviation or more.

The value of testing colour vision depends on the sensitivity and specificity of the test, and this is shown in Fig 2 for the tritan threshold. The discrimination obtained between normal and glaucomatous eyes varied with the colour contrast, which is considered to be abnormal. Fig 2 shows the effect of altering the criterion value in intervals of $1 \%$ from 4 to $11 \%$. If the low threshold is considered to separate the normal from the abnormal, there were many false posi-
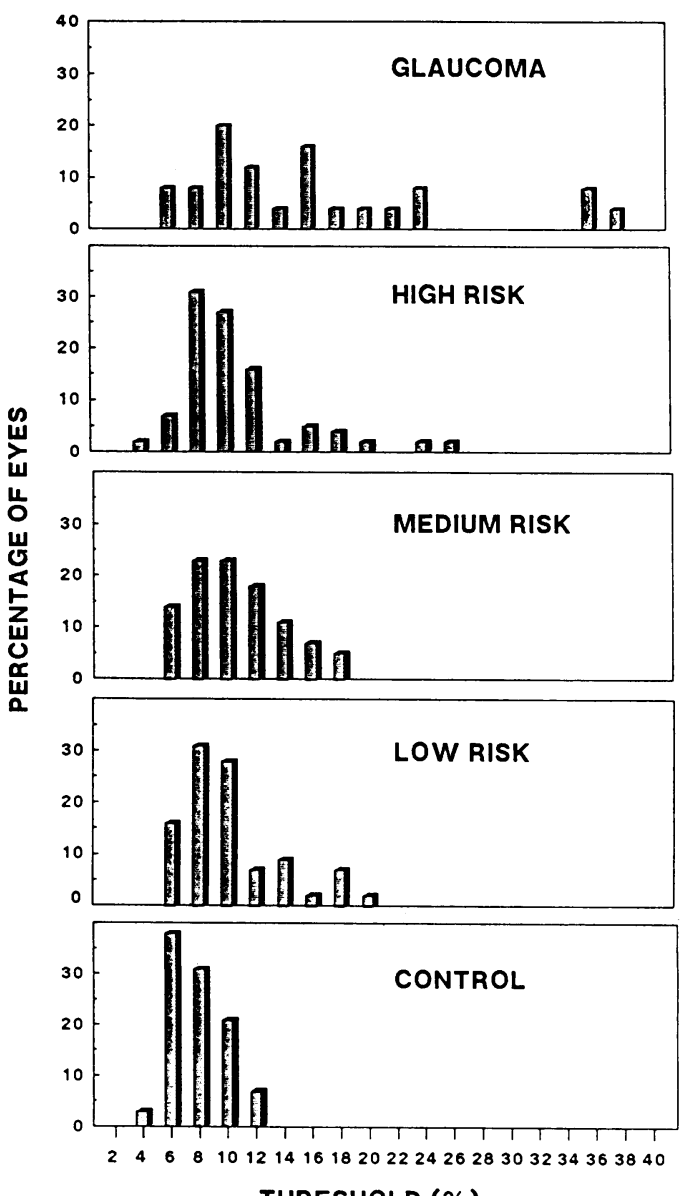

THRESHOLD (\%)

Figure 1 Distribution of tritan thresholds of colour contrast sensitivity. Maximum colour contrast consisted of a background of $(X=0 \cdot 443, Y=0 \cdot 445)$ on which letters were superimposed with a colour of $(X=0 \cdot 302, Y=0 \cdot 223)$ 
NORMALS

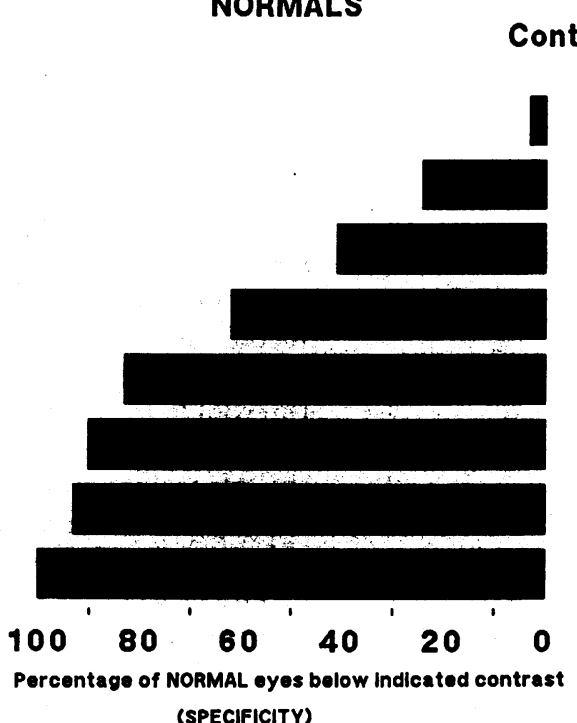

\section{GLAUCOMA}

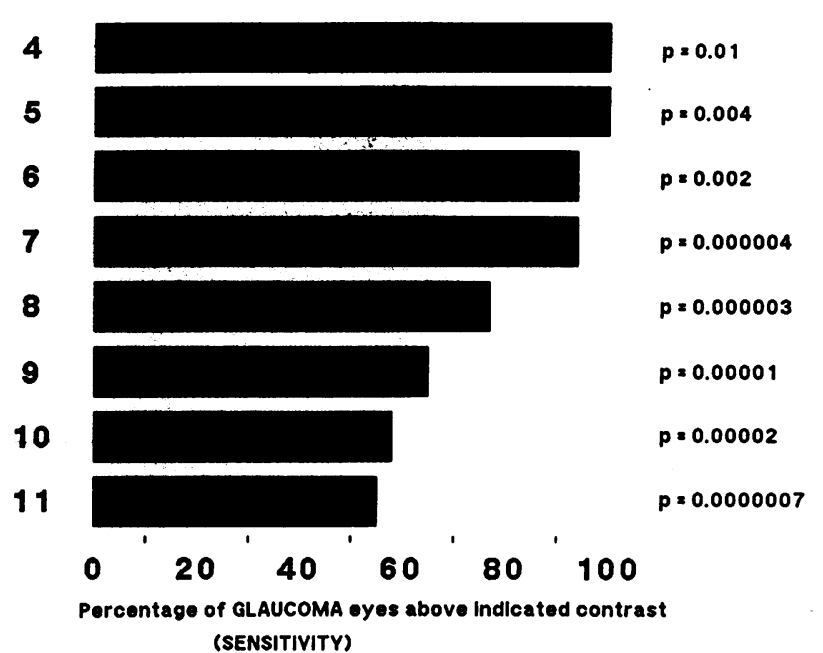

Figure 2 Analysis of results of colour contrast sensitivity in the tritan axis. Numbers in the middle of the graph represent the range of modulation contrast in which there was overlap between glaucomatous and normal eyes. Bars to the right show percentage of glaucomatous eyes above the indicated contrast. Bars to the left shows the percentage of normal eyes below the indicated contrast. Values of p for each cut-off are from Fisher's exact test. $8 \%$ gives the best combination of specificity and sensitivity.

tives, but all glaucoma patients were considered abnormal. If the criterion is set high, then there were very few false positives, but nearly half the glaucoma patients were false negatives. Thus the length of the bars on the left in Fig 2 represent the specificity, and the bars to the right the sensitivity. Values of $p$ (Fisher's exact test) are indicated for each cut-off level. The best sensitivity and specificity are obtained with a cutoff of $8 \%$, which is similar to the previously determined upper limit of normal. Statistically the results are highly significant. However, $23 \%$ of glaucomatous eye would be considered normal by this criterion. This result is not due to the imprecision in the test. For low and medium risk patients who were seen three or more times the difference between tests was less than $1 \%$. If this is taken as an index of test-retest variability (and see Fig 4), there was during the investigation a progressive loss of sensitivity in glaucoma patients, whose mean threshold increased by $2 \%$ between the first and second visit and by $>1 \%$ between the second and the third.

The tritan thresholds for all visits are shown in Table 2. Since patients were recruited during the period of the investigation, the number of eyes progressively decreased with visit number, which precludes comparison between columns.

Table 2 Mean tritan thresholds for all classes - four visits

\begin{tabular}{lllll}
\hline Class & 1st visit & 2nd visit & 3rd visit & 4th visit \\
\hline Glaucoma & & & & \\
$\quad$ Mean & 19 & 18 & 14 & 14 \\
SD & 16 & 14 & 7 & 7 \\
Number & 32 & 24 & 16 & 10 \\
High risk & 10 & 10 & 11 & 11 \\
Mean & 4 & 3 & 3 & 4 \\
SD & 59 & 44 & 44 & 30 \\
Number & 59 & & 9 & 10 \\
Medium risk & 10 & 9 & 9 & 2 \\
$\quad$ Mean & 4 & 5 & 4 & 23 \\
SD & 56 & 43 & 31 & 10 \\
Number & 56 & 10 & 10 & 10 \\
Low risk & 9 & 3 & 4 & 5 \\
Mean & 3 & 3 & 34 & 21 \\
ND & 54 & 49 & & \\
\hline
\end{tabular}

However, if the mean of thresholds of the eyes seen on three visits are considered $(n=16)$, the variation between the three visits is less than $2 \%$.

Because some glaucomatous eyes have normal colour vision, we examined the results to see if any other clinical features were related to this finding. However, neither age, sex distribution, cup/disc ratio, or distance of the field defect from fixation or its size is related to a loss of central colour vision (data not illustrated). The incidence of congenital tritan defects in the population is so low ( $<1$ in a million) that this need not be considered.

\section{HIGH RISK OCULAR HYPERTENSIVES}

Fifty nine eyes with high risk hypertension were tested on the first visit, 44 were tested on two occasions, 41 three times, and 30 on four occasions (Table 1). The thresholds obtained in the three colour confusion lines for high risk $\mathrm{OH}$ patients on the first visit are shown in Table 1. Thresholds were lower than glaucomatous eyes' thresholds but slightly above the mean threshold of the control group. Fig 1 shows the distribution of thresholds in the tritan axis in this group. Different criteria of abnormality were again considered, and Fig 3 shows the number of patients who scored abnormal and the number of controls who scored normal for each level. Values of $p$ (Fisher's exact test) are indicated. If the $8 \%$ level is taken as cut-off (as in the glaucoma group) $50 \%$ of eyes would score abnormal. Although the $p$ values are significant, they are much larger than for glaucoma patients. The variation in thresholds for successive visits in high risk patients was small (Fig 4). Thus the test-retest variation contributed almost nothing to the variability of the results.

The mean threshold of all eyes in each visit (Table 2) was nearly the same, and the variation of mean threshold of eyes seen on three visits $(n=$ 41) confirms a variation of less than $1 \%$. Thus comparison of Figs 2 and 3 suggests that the 


\section{Contrast (\%)}
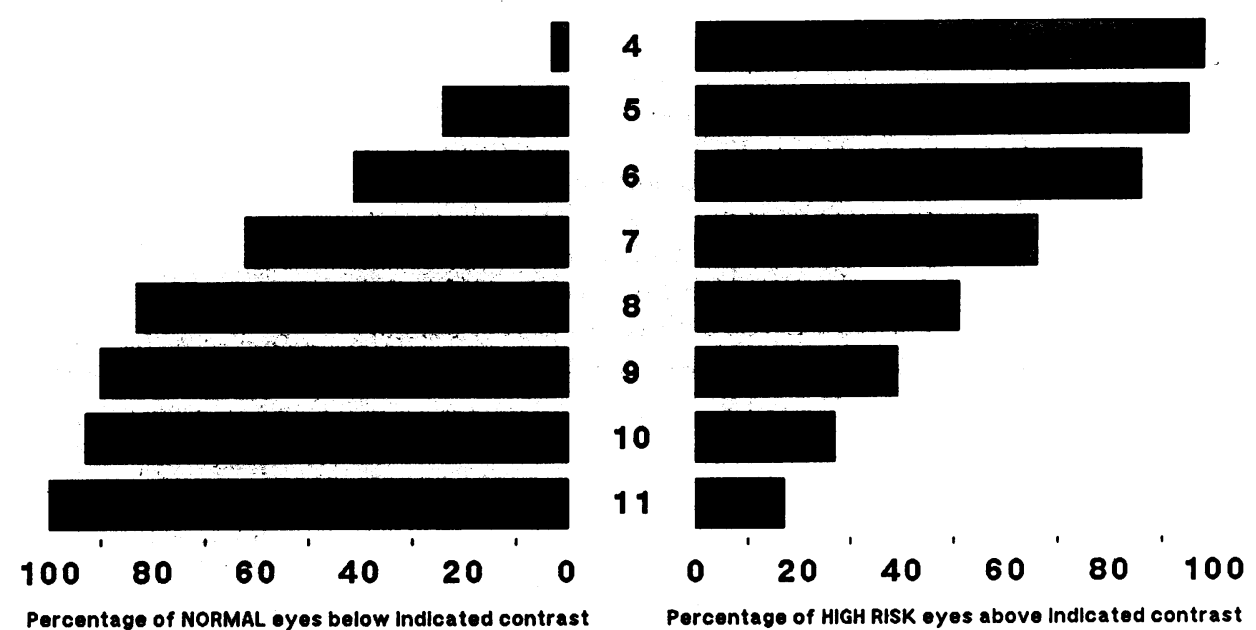

Figure 3 Analysis of results of $C C S$ in the tritan axis of high risk $O H$ patients. Numbers in the middle of the graph represent the range of modulation contrast over which there was overlap between high risk $\mathrm{OH}$ and normal eyes. Bars to the right shows the percentage of high risk $\mathrm{OH}$ eyes above indicated contrast. Bars to the left shows the percentage of normal eyes below the indicated contrast. Values of $p$ for each cut-off level are from Fisher's exact test.

specificity and sensitivity were similar for the two groups.

\section{MEDIUM RISK GROUP}

Fifty six eyes with medium risk hypertension were tested in the first visit, 43 were tested in two visits, 32 in three, and 23 in four visits. The means of thresholds obtained in the three colour confusion lines for medium risk $\mathrm{OH}$ patients on the first visit are shown in Table 2. Tritan thresholds were slightly more impaired than protan or duetan and similar to those from high risk patients. The distribution of tritan thresholds almost overlapped that of normal persons (Fig 1).
LOW RISK GROUP

Fifty four eyes in this group were tested in the first visit, 49 were tested on two occasions, 34 in three, and 21 in four. Table 1 shows the mean thresholds for each colour obtained in the low risk eyes on the first visit. Mean thresholds are not significantly different from those of the normal persons and the other hypertension groups. The distribution of results of eyes with low risk was similar to that obtained in the control group (Fig 1). With $8 \%$ taken as a cut-off $43 \%$ of low risk would be considered abnormal. The measurements of tritan thresholds for all visits are given in Table 2. Variation of mean thresholds of eyes seen on three visits $(n=34)$ was within $1 \%$ (Table 2).

\section{HIGH RISK OH GROUP}

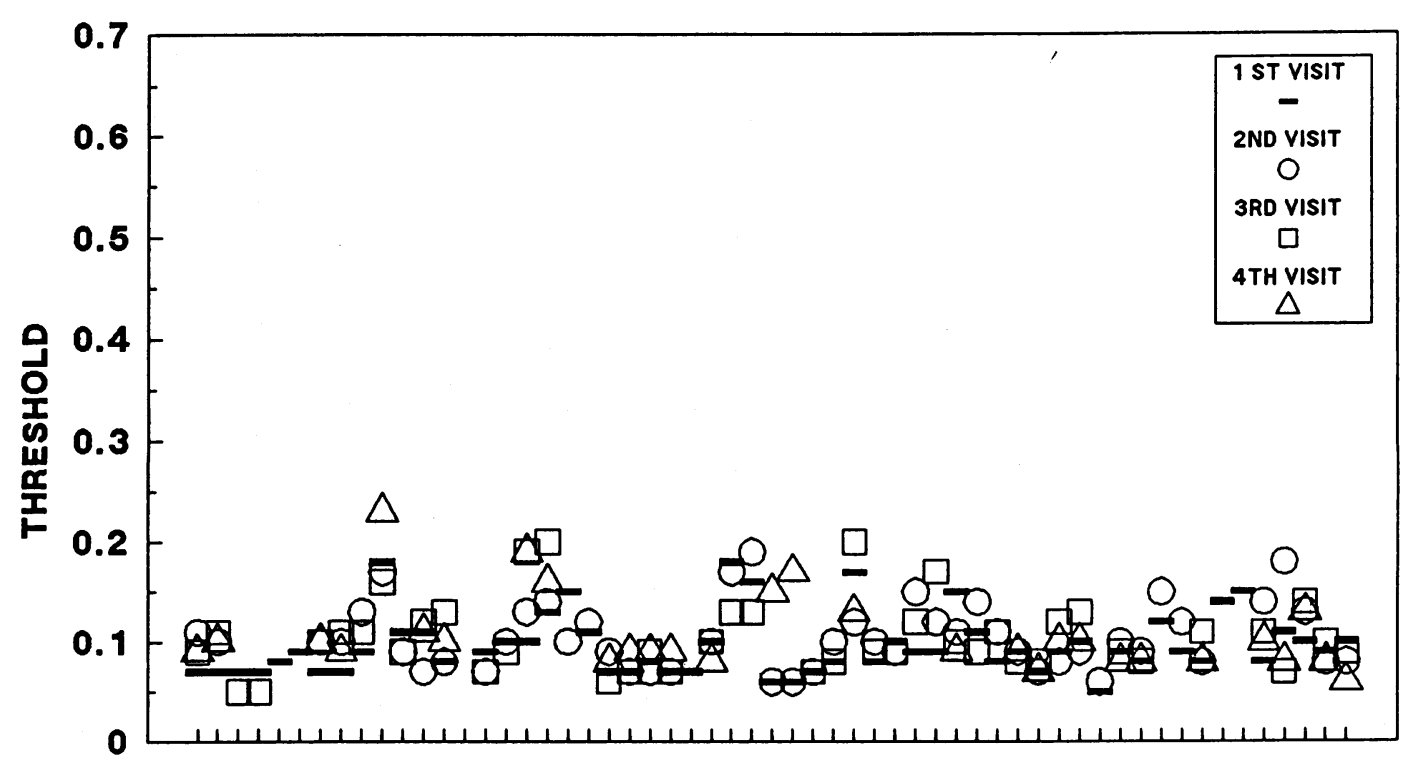

Figure 4 Results of CCS in high risk group at four visits (tritan axis). Note that there is slight deviation towards higher values. Each division on the $x$ axis represents a different subject. 
CORRELATION BETWEEN TRITAN COLOUR VISION AND THE PATTERN ERG

The relationship between PERG amplitude (from the peak of P50 to the trough of N95) was investigated for all patients with $\mathrm{OH}$ and glaucoma. The results (not illustrated) showed a scattergram with little apparent correlation $(\mathrm{r}=$ 0.31 for 583 pairs of measurement). However, in 139 cases both tests gave normal results, and in 202 both gave abnormal results. The colour test was normal in 77 cases with abnormal PERG, and abnormal colour vision occurred in 161 cases with a normal PERG. This result is very similar to that of Trick et al, ${ }^{26}$ who used the Lanthony desaturated D15 colour test.

\section{Discussion}

We studied a large population of ocular hypertensives at different risks of conversion to glaucoma, glaucoma at an early stage, and normotensive glaucoma patients every six months during a two-year period. Combining all the different $\mathrm{OH}$ classes, we found the mean tritan threshold to be greater than for the control group, and $32 \%$ of all hypertensive eyes had a threshold elevation which deviated more than 2 SD from the mean of the control group. These $\mathrm{OH}$ eyes with abnormal colour vision may represent the fraction of $\mathrm{OH}$ prone to develop glaucoma. This has been demonstrated in a prospective study. ${ }^{9}$ Thresholds in protan and deutan axes are also greater in high risk than in low risk $\mathrm{OH}$, but the difference is less evident than for the tritan results. A significant reduction in colour vision was demonstrated in glaucoma patients for all three colour axes.

However, a considerable overlap exists between the patients and the control population. The value of 2 SDs above the control population did not completely discriminate the glaucoma patients from controls. Our results are in agreement with others in that some glaucomatous eyes never develop loss of central colour vision. Though our system is much more sensitive than other colour vision tests, the proportion of normal results is the same as previously reported. The results of this investigation contrast with that of another study from our clinic, which shows that contrast sensitivity when measured in the peripheral retina to luminance contrast, ${ }^{25}$ and especially colour contrast, ${ }^{27}$ is universally reduced in an area within $10^{\circ}$ to $25^{\circ}$ from the fovea in all glaucomatous eyes and in $50 \%$ of high risk hypertensive eyes.

Thus there seem to be two patterns of colour vision loss in glaucoma. In one the defect stops short of the macula, while in the other it is diffuse. Thus our data support the concept of two distinct types of mechanism of damage, one leading to diffuse and the other to focal damage in the optic nerve. ${ }^{2829}$ The poor correlation between central colour vision and PERG findings can also be explained on the basis of two types of glaucomatous loss, for the PERG is largely produced by peripheral retinal stimulation and by a system that is not responsive to colour contrast. ${ }^{30}$
Falcäo-Reis acknowledges a scholarship from Calouste Gulbenkian Foundation, Lisbon. The authors would like to thank Professor Joaquim Maia for the statistical support.

Colleagues, particularly $\mathrm{Mr} \mathrm{R} A$ Hitchings and $\mathrm{Mr} \mathrm{E}$ O'Donoghue, have been most generous in supplying information about patients, and sending them to the Electrodiagnostic Departabout patients, and sending them to the Electrodiagnostic Department for testing. The authors also thank all the staff of the
Electrodiagnostic Department for their collaboration and help during the course of this study.

1 Sample PA, Weinreb RN, Boyton RM. Acquired dyschromatopsia in glaucoma. Surv Ophthalmol 1986; 31: 54-64.

2 Lakowski R, Bryett J, Drance SM. A study of colour vision in ocular hypertension. Can $\mathcal{F}$ Ophthalmol 1972; 7: 86-95.

3 Drance SM, Airaksinen PJ. Signs of early damage in open angle glaucoma. In: Weinstein $\mathrm{GW}$, ed. Open angle glaucoma. (Contemporary issues in ophthalmology, 3.) New York: Churchill Livingstone, 1986: 17-30.

4 Drance SM. The early structural and functional disturbances of chronic open-angle glaucoma. Ophthalmology 1985; 92. 853-7.

5 Biersdorf WR. The Davidson and Hemmendiger colour rule as a colour vision screening test. Arch Ophthalmol 1977; 95: 134-8.

6 Breton ME, Tansley BW. Improved colour test results with large-field viewing in dichromats. Arch Ophthalmol 1985; 103: $1490-4$

7 Farnsworth D. The Farnsworth-Munsell 100-hue dichotomous tests for colour vision. $\mathcal{F}$ Opt Soc Am 1943; 33: 568-76

8 Drance SM, Lakowski R, Schulzer M, Douglas GR. Acquire color vision changes in glaucoma. Use of 100-hue test and Pickford anomaloscope as predictors of glaucoma field change. Arch Ophthalmol 1981; 99: 829-31.

9 Verriest G, Buyssens A, Vanderdonck R. Etude quantitative de l'effet de l'exercice sur les résultats de quelques tests de discrimination chromatique: une diminution non selective
du niveau d'un éclairage. $C R$ Rev Optique (Paris) 1963; 42: du nivea 10 .

10 Hart WM. Acquired dyschromatopsias. Surv Ophthalmol 1987; 32: 10-31

11 Pokorny J, Smith VC, Verriest G, Pinckers AJLG. Congenital and acquired color vision defects. New York: Grune and Stratton, 1979: 174-81.

12 Logan N, Anderson DR. Detecting early glaucomatous visual field changes with a blue stimulus. Am $\mathcal{F}$ Ophthalmol 1983; 95: 432-4.

13 Hart WM, Burde RM. Color contrast perimetry. The spatial distribution of color defects in optic nerve and retinal diseases. Ophthalmology 1985; 92: 768-76.

14 Hart WM, Silverman SE, Trick GL, Nesmer R, Gordon MO Glaucomatous visual field damage. Invest Ophthalmol Vis $S_{c}$ 1990; 31: 359-67.

15 Arden GB, Gunduz K, Perry S. Colour vision testing with a computer graphics system: preliminary results. Doc Ophthalmol 1988; 69: 167-74.

16 Arden GB, Gunduz K, Perry S. Colour vision testing with a computer system. Clin Vision Sci 1988; 2: 203

17 Potts M, Fells P, Falcao-Reis F, Arden GB. Colour contrast sensitivity, pattern electroretinogram and cortical visual evoked potentials in dysthyroid optic neuropathy. Invest Ophthalmol Vis Sci 1990; 31 (suppl): 189.

18 Gunduz K, Arden GB, Perry S, Weinstein GW, Hitchings R. Color vision defects in ocular hypertension and glaucoma: quantification with a computer-driven color television system. Arch Ophthalmol 1988; 108: 929-35.

19 Armaly MF, Krueger DE, Maunder L, et al. Biostatistica analysis of the collaborative glaucoma study. I. Summary report of the risk factors for glaucomatous visual-field defects. Arch Ophthalmol 1980; 98: 2163-71.

20 Motolko M, Drance SM. Features of the optic disc in preglaucomatous eyes. Arch Ophthalmol 1981; 99: 1992-4.

21 Pederson JE, Anderson DR. The mode of progressive disc cupping in ocular hypertensive and glaucoma. Arch Ophthalmol 1980; 98: 490-5.

22 Yablonsky M, Zimmerman T, Kass M, Becker B. Prognostic significance of optic disk cupping in ocular hypertensive significance of optic disk cupping in ocula

23 Arden GB, Vaegan. Electroretinograms evoked in man by local uniform and pattern stimulation. $\mathcal{f}$ Physiol (Lond) 1983; 341: 85-104.

24 Weinstein GW, Arden GB, Hitchings RA, et al. The pattern electroretinogram (PERG) in ocular hypertension and glaucoma. Arch Ophthalmol 1988; 106: 923-8.

25 Falcao-Reis FM, O'Donoghue E, Buceti R, Hitchings $R$, Arden GB. Peripheral contrast sensitivity in glaucoma and ocular hypertension. Br F Ophthalmol 1990; 74: 712-6.

26 Trick GL, Nesher R, Cooper DG, Kolker AE, Bicckler-Bluth $M$. Dissociation of visual deficits in ocular hypertension. M. Dissociation of visual deficits in ocular
Invest Ophthalmol Vis Sci 1988; 29: 1486-91.

27 Yu Tak C, Falcao-Reis F, Spileers W, Arden GB. A rapid screening test for glaucoma. Invest Ophthalmol Vis Sci (in press).

28 Yamazaki Y, Lakowski R, Drance SM. A comparison of the blue color mechanism in high- and low-tension glaucoma. Ophthalmology 1989; 96: 12

29 Gloster J. Incidence of optic disc haemorrhages in chronic open-angle glaucoma and ocular hypertension. $\mathrm{Br} \mathcal{J}$ Ophthalmol 1981; 65: 452-6.

30 Berninger TA, Arden GB, Hogg CR, Frumkes T. Separable evoked retinal and cortical potentials from each major visual pathway: preliminary results. $\mathrm{Br} \mathcal{F}$ Ophthalmol 1989; 73 502-11. 